\title{
Spherically symmetric analytic solutions and naked singularities in Einstein-Aether theory
}

\author{
R. Chan ${ }^{1, a}$, M. F. A. da Silva ${ }^{2, b}$, V. H. Satheeshkumar ${ }^{3, c}$ \\ ${ }^{1}$ Coordenação de Astronomia e Astrofísica, Observatório Nacional (ON), Rio de Janeiro, RJ 20921-400, Brazil \\ 2 Departamento de Física Teórica, Universidade do Estado do Rio de Janeiro (UERJ), Rio de Janeiro, RJ 20550-900, Brazil \\ ${ }^{3}$ Departamento de Física, Universidade Federal do Estado do Rio de Janeiro (UNIRIO), Rio de Janeiro, RJ 22290-240, Brazil
}

Received: 9 January 2021 / Accepted: 6 April 2021 / Published online: 13 April 2021

(C) The Author(s) 2021

\begin{abstract}
We analyze all the possible spherically symmetric exterior vacuum solutions allowed by the Einstein-Aether theory with static aether. We show that there are three classes of solutions corresponding to different values of a combination of the free parameters, $c_{14}=c_{1}+c_{4}$, which are: $0<c_{14}<2, c_{14}<0$, and $c_{14}=0$. We present explicit analytical solutions for $c_{14}=3 / 2,16 / 9,48 / 25,-16$ and 0 . The first case has some pathological behavior, while the rest have all singularities at $r=0$ and are asymptotically flat spacetimes. For the solutions $c_{14}=16 / 9,48 / 25$ and -16 we show that there exist no horizons, neither Killing horizon nor universal horizon, thus we have naked singularities. This characteristic is completely different from general relativity. We briefly discuss the thermodynamics for the case $c_{14}=0$ where the horizon exists.
\end{abstract}

\section{Introduction}

The Lorentz invariance is an exact symmetry of special relativity, quantum field theories and the standard model of particle physics, and also a local symmetry in freely falling inertial frames in general relativity [1]. The Lorentz violation in matter interactions is highly constrained by several precision experiments, see [2] for the latest example, while similar studies in gravity are not as well explored. With this motivation, Jacobson and collaborators introduced and analyzed a general class of vector-tensor theories called the EinsteinAether (EA) theory [3-7].

The first paper to investigate spherical static vacuum solutions in the EA theory was presented by Eling and Jacobson in 2006 [8]. In that paper, the authors found a family of ana-

\footnotetext{
a e-mail: chan@on.br (corresponding author)

b e-mail: mfasnic@gmail.com

c e-mail: vhsatheeshkumar@gmail.com
}

lytical solutions for the metric functions, up to the inversion of a transcendental function, assuming an aether vector proportional to the timelike Killing field. These solutions do not depend on the parameters $c_{2}$ and $c_{3}$ of the EA theory but only depend on the combination $c_{1}+c_{4}$, which for simplicity we denote as $c_{14}$. such that $c_{1}+c_{4}<2$. For $c_{1}+c_{4}>2$ the coupling constant $G$ becomes negative, implying that the gravity is repulsive, and for $c_{1}+c_{4}=0$ this one is exactly the Newtonian gravitational constant. The authors have shown when $c_{1}+c_{4}=0$ the Schwarzschild solution can be obtained and also explored some solutions for different choices of $0 \leq c_{1}+c_{4}<2$. In another paper, Eling, Jacobson and Miller [9] studied a perfect fluid, in order to model a neutron star, and consider the vacuum solution given in the previous paper [8] for any $c_{1}+c_{4}<2$. Almost the complete literature on black holes in EA theory can be found in the papers [10-30].

Since this article deals with naked singularities, some introductory remarks are in order. In 1965, Roger Penrose proved the first modern singularity theorem [31] for which he is recognized with a Nobel Prize in physics last year [32]. In this paper, Penrose introduced the notion of closed trapped surface for the first time to prove the gravitational collapse in GR inevitably leads to singularities. The most important physical consequence of the singularity theorem is to know whether these singularities can be observed. Typically the singularities that arise in the solutions of Einstein field equations are hidden within event horizons. The singularities that are not hidden inside the event horizon are called naked singularities. The Cosmic Censorship Conjecture (CCC), introduced by Penrose in 1969 [33], does not permit singularities void of an event horizon as the end state of gravitational collapse for generic regular initial data and for a suitable matter system. Although there is no satisfactory mathematical formulation or the proof of CCC, there are many examples of dynamical collapse models which lead to 
a black hole or a naked singularity as the collapse end state, depending on the nature of the initial data (see e.g. [34-41] and references therein). Two major themes in this context are either mathematically prove or disprove the CCC or provide counter examples. Naked singularities are of current interest because they have observational properties quite different from a black hole. Besides, theoretically these regions of extreme gravity might have some hints of quantum gravity. If observed, they will provide exciting laboratory for the discovery of new physics. Most up-to-date account on naked singularities can be found in [42]. Some of the observational consequences of naked singularities explored recently can be found in the references [43-48].

The paper is organized as follows. The Sect. 2 briefly outlines the EA theory, whose field equations are solved for a general spherically symmetric static metric in Sect. 3. In Sects. 4 and 5 we present the explicit analytical solutions. We briefly discuss black hole thermodynamics in Sect. 6 for the case $c_{14}=0$ where the horizon exists. We summarize our results in Sect. 7.

\section{Field equations in the EA theory}

The general action of the EA theory is given by

$$
S=\frac{1}{16 \pi G} \int \sqrt{-g}\left(R+L_{\text {aether }}+L_{\text {matter }}\right) d^{4} x,
$$

where, the first term defined by $R$ is the usual Ricci scalar, and $G$ the EA coupling constant. The second term, the aether Lagrangian is given by

$L_{\text {aether }}=\left[-K^{a b}{ }_{m n} \nabla_{a} u^{m} \nabla_{b} u^{n}+\lambda\left(g_{a b} u^{a} u^{b}+1\right)\right]$,

where the tensor $K^{a b}{ }_{m n}$ is defined as

$K^{a b}{ }_{m n}=c_{1} g^{a b} g_{m n}+c_{2} \delta_{m}^{a} \delta_{n}^{b}+c_{3} \delta_{n}^{a} \delta_{m}^{b}-c_{4} u^{a} u^{b} g_{m n}$,

being the $c_{i}$ dimensionless coupling constants, and $\lambda$ a Lagrange multiplier enforcing the unit timelike constraint on the aether, and

$\delta_{m}^{a} \delta_{n}^{b}=g^{a \alpha} g_{\alpha m} g^{b \beta} g_{\beta n}$.

Finally, the last term, $L_{\text {matter }}$ is the matter Lagrangian, which depends on the metric tensor and the matter field.

In the weak-field, slow-motion limit EA theory reduces to Newtonian gravity with a value of Newton's constant $G_{\mathrm{N}}$ related to the EA coupling constant $G$ in the action (1) by [11],

$G=G_{N}\left(1-\frac{c_{14}}{2}\right)$.
Here, the constant $c_{14}$ is defined as

$c_{14}=c_{1}+c_{4}$.

Note that if $c_{14}=0$ the EA coupling constant $G$ becomes the Newtonian coupling constant $G_{N}$, without necessarily imposing $c_{1}=c_{4}=0$. For $c_{14}>2$ the coupling constant $G$ becomes negative, implying that the gravity is repulsive. The coupling constant vanishes when $c_{14}=2$, this in turn renders the action undefined. Thus, physically interesting region is $c_{14}<2$ where the Newtonian limit can be recovered.

The field equations are obtained by extremizing the action with respect to independent variables of the system. The variation with respect to the Lagrange multiplier $\lambda$ imposes the condition that $u^{a}$ is a unit timelike vector, thus

$g_{a b} u^{a} u^{b}=-1$,

while the variation of the action with respect $u^{a}$, leads to [11]

$\nabla_{a} J^{a}{ }_{b}+c_{4} a_{a} \nabla_{b} u^{a}+\lambda u_{b}=0$,

where,

$J^{a}{ }_{m}=K^{a b}{ }_{m n} \nabla_{b} u^{n}$,

and

$a_{a}=u^{b} \nabla_{b} u_{a}$.

The variation of the action with respect to the metric $g_{m n}$ gives the dynamical equations,

$G_{a b}^{\text {Einstein }}=T_{a b}^{a e t h e r}+8 \pi G T_{a b}^{\text {matter }}$,

where

$$
\begin{aligned}
G_{a b}^{\text {Einstein }}= & R_{a b}-\frac{1}{2} g_{a b} R, \\
T_{a b}^{\text {aether }}= & \left.\nabla_{c}\left[J^{c}{ }_{(a} u_{b)}+u^{c} J_{(a b)}-J_{(a}{ }^{c} u_{b}\right)\right] \\
& -\frac{1}{2} g_{a b} J^{c}{ }_{d} \nabla_{c} u^{d}+\lambda u_{a} u_{b} \\
& +c_{1}\left[\nabla_{a} u_{c} \nabla_{b} u^{c}-\nabla^{c} u_{a} \nabla_{c} u_{b}\right]+c_{4} a_{a} a_{b}, \\
T_{a b}^{\text {matter }}= & \frac{-2}{\sqrt{-g}} \frac{\delta\left(\sqrt{-g} L_{\text {matter }}\right)}{\delta g_{a b}} .
\end{aligned}
$$

Later, when we solve the field equations (11), we do take into consideration the equations (7)-(10) in the process of simplification. Thus in the paper (as in the equations (20)(23) below) we seem to solve only the dynamical equations, but in fact we are also solving the equations arising from the variations of the action with respect $\lambda$ and $u^{a}$. 
In a more general situation, the Lagrangian of GR theory is recovered, if and only if, the coupling constants are identically null, e.g., $c_{1}=c_{2}=c_{3}=c_{4}=0$, considering the Eqs. (3) and (7).

\section{Spherical solutions of EA field equations}

We start with the most general spherically symmetric static metric

$d s^{2}=-e^{2 A(r)} d t^{2}+e^{2 B(r)} d r^{2}+r^{2} d \theta^{2}+r^{2} \sin ^{2} \theta d \phi^{2}$.

In accordance with Eq. (7), the aether field is assumed to be unitary and timelike, chosen as

$u^{a}=\left(e^{-A(r)}, 0,0,0\right)$.

This choice is not the most general and is restricted to the scenario where aether is static. The aether must tip in a black hole solution as it cannot be timelike be aligned with the null Killing vector on the horizon. As that is not the case with our choice, our solutions are valid only outside the Killing horizon. This is good enough for solar system tests and even for astrophysical solutions to describe the exterior spacetime to a source.

The timelike Killing vector of the metric (13) is giving by

$\chi^{a}=(-1,0,0,0)$.

The Killing and the universal horizon [49,50] are obtained finding the largest root of

$\chi^{a} \chi_{a}=0$

and

$\chi^{a} u_{a}=0$

respectively, where $\chi^{a}$ is the timelike Killing vector. In our case,

$\chi^{a} \chi_{a}=-e^{2 A(r)}$,

$\chi^{a} u_{a}=e^{A(r)}$.

For the general spherically symmetric metric (13), we compute the different terms in the field equations (12) below.

$$
\begin{aligned}
G_{t t}^{\text {aether }}= & -\frac{e^{2(A-B)}}{2 r^{2}}\left[c_{14}\left(-2 r^{2} A^{\prime} B^{\prime}+r^{2} A^{\prime 2}+2 r^{2} A^{\prime \prime}+4 r A^{\prime}\right)\right. \\
& \left.\times-4 r B^{\prime}-2 e^{2 B}+2\right]=0, \\
G_{r r}^{\text {aether }}= & -\frac{1}{2 r^{2}}\left(c_{14} r^{2} A^{\prime 2}+4 r A^{\prime}-2 e^{2 B}+2\right)=0,
\end{aligned}
$$

$$
\begin{aligned}
G_{\theta \theta}^{\text {aether }}= & \frac{r}{2 e^{2 B}} \\
& \times\left(2 r A^{\prime \prime}-2 r A^{\prime} B^{\prime}+2 A^{\prime}-2 B^{\prime}-\left(c_{14}-2\right) r A^{\prime 2}\right)=0,
\end{aligned}
$$

$G_{\phi \phi}^{a e t h e r}=G_{\theta \theta}^{\text {aether }} \sin ^{2} \theta$,

where $G_{\mu \nu}^{a e t h e r}=G_{\mu \nu}^{\text {Einstein }}-T_{\mu \nu}^{a e t h e r}$. In order to identify eventual singularities in the solutions, it is useful to calculate the Kretschmann scalar invariant K. For the metric (13), it is given by

$$
\begin{aligned}
K= & \frac{4}{r^{4} e^{4 B}}\left(2 B^{\prime 2} r^{2}+e^{4 B}-2 e^{2 B}+1+2 A^{\prime 2} r^{2}+r^{4} A^{\prime \prime 2}+2 r^{4} A^{\prime \prime} A^{\prime 2}\right. \\
& \left.-2 r^{4} A^{\prime \prime} B^{\prime} A^{\prime}+r^{4} A^{\prime 4}-2 r^{4} A^{\prime 3} B^{\prime}+r^{4} B^{\prime 2} A^{\prime 2}\right)
\end{aligned}
$$

Before we proceed, we simplify the field equations. Substituting the field Eq. (21) into (20) we can eliminate the term $e^{2 B}$ and find

$c_{14}\left(2 r A^{\prime \prime}-2 r A^{\prime} B^{\prime}+4 A^{\prime}\right)-4 A^{\prime}-4 B^{\prime}=0$.

We have two field Eqs. (22) and (25), to solve. Using these two, we can eliminate $A^{\prime \prime}$ only when $c_{14} \neq 0$ and obtain the following,

$\left(c_{14}-2\right)\left(B^{\prime}+A^{\prime}+\frac{1}{2} r c_{14} A^{\prime 2}\right)=0$.

We can obtain another equation when $c_{14}=0$,

$r A^{\prime \prime}+2 r A^{\prime 2}+2 A^{\prime}=0$

From the Eq. (26) we can note that there are two possibilities: $c_{14}=2$ and $c_{14} \neq 2$, of which $c_{14}=2$ is not permitted. As we mentioned previously, $c_{14}>2$ implies that the gravity is repulsive and $c_{14}<0$ means gravity is stronger. Thus, both mathematical and physical justifications lead us to three possible cases of interest: (i) $0<c_{14}<2$ (ii) $c_{14}<0$ (iii) $c_{14}=0$. We now analyze these three possible cases in detail.

In order to find explicit solutions for the metric functions we used the Maple 16 algebraic software following the algorithm: we have made a survey of all possible explicit solutions imposing $c_{14}=m / n$, where $m$ and $n$ are integer constants in the interval $0<n<100$ and $0<m<100$ varying in step of one and imposing that $0<c_{14}<2$ or $c_{14}<0$. This survey has taken several hours of computational time in an old CPU generation computer (Intel Duo Core).

\section{Solutions for $0<\mathrm{c}_{14}<2$ and $\mathrm{c}_{14}<0$}

The non-trivial solutions in EA theory exist for $0<c_{14}<2$ which help us understand the physical effects of aether. As we mentioned earlier, we have two field Eqs. (22) and (25), 
to solve. We first use (25) to express $B^{\prime}$ as

$$
\left(2+c_{14} r A^{\prime}\right) B^{\prime}=c_{14} r A^{\prime \prime}+2 c_{14} A^{\prime}-2 A^{\prime} .
$$

From this equation we can notice that if $2+c_{14} r A^{\prime}=0$, we would get $A^{\prime}=-\frac{2}{c_{14} r}$ which would not lead to any solution. Therefore, we proceed by assuming $2+c_{14} r A^{\prime} \neq 0$. Substituting $B^{\prime}$ into (22) we have also

$\left(c_{14}-2\right)\left(2 r A^{\prime \prime}+4 r A^{\prime 2}+4 A^{\prime}+r^{2} c_{14} A^{\prime 3}\right)=0$.

From the Eqs. (26) and (29), we have

$B^{\prime}+A^{\prime}+\frac{1}{2} r c_{14} A^{\prime 2}=0$,

and

$2 r A^{\prime \prime}+4 r A^{\prime 2}+4 A^{\prime}+r^{2} c_{14} A^{\prime 3}=0$.

Substituting $B^{\prime}$ and $A^{\prime \prime}$ from these equations into the field Eqs. (20)-(21) we get only an unique equation

$r^{2} c_{14} A^{\prime 2}+4 r A^{\prime}-2 e^{2 B}+2=0$.

Solving Eq. (32) for B we have

$B=\frac{1}{2} \ln \left(\frac{1}{2} r^{2} A^{\prime 2} c_{14}+2 A^{\prime} r+1\right)$.

Now, differentiating (33) and substituting into (30) we get (31) which implies consistency.

\subsection{Solution for $\mathbf{c}_{14}=\mathbf{3} / \mathbf{2}$}

Solving the Eq. (31) we can obtain an analytical solution for $A^{\prime}$ for a particular case $c_{14}=3 / 2$. Making a transformation $u(r)=A^{\prime}$ we can reduce the second order differential equation in $A$ into a first order differential equation in $u$, thus

$2 r u^{\prime}+4 r u^{2}+4 u+\frac{3}{2} r^{2} u^{3}=0$

This equation is easily integrated giving three solutions which one is real and two are imaginaries. The real one is given by

$$
\begin{gathered}
u=A^{\prime}=-\frac{2}{3 r}\left\{1-\frac{\left[r^{2}\left(\sqrt{27 \beta^{2}-r^{2}}+3 \sqrt{3} \beta\right)\right]^{\frac{1}{3}}}{\sqrt{27 \beta^{2}-r^{2}}}\right. \\
\left.+\frac{r^{2}}{\sqrt{27 \beta^{2}-r^{2}}\left[r^{2}\left(\sqrt{27 \beta^{2}-r^{2}}+3 \sqrt{3} \beta\right)\right]^{\frac{1}{3}}}\right\},
\end{gathered}
$$

where $\beta$ is an arbitrary integration constant.

Since $r^{2}<27 \beta^{2}$, we notice that this solution is not real in all spacetime but only in the region near to the center, meaning that this solution can not represent the exterior of a source. Thus, it will not be analyzed in more detail.

\subsection{Solution for $\mathbf{c}_{\mathbf{1 4}}=\mathbf{1 6} / \mathbf{9}$}

Solving the Eq. (31) we can obtain an analytical solution for $A^{\prime}$ for another particular case $c_{14}=16 / 9$. Making a transformation $u(r)=A^{\prime}$ we can reduce the second order differential equation in $A$ into a first order differential equation in $u$, thus

$2 r u^{\prime}+4 r u^{2}+4 u+\frac{16}{9} r^{2} u^{3}=0$.

This equation is easily integrated to get two solutions given by

$u=A^{\prime}=-\frac{3}{4}\left(\frac{1}{r}+\frac{\delta}{\sqrt{(r-8 \alpha) r}}\right)$,

where $\alpha$ is an arbitrary integration constant and $\delta= \pm 1$. Integrating once this equation and assuming the new arbitrary constant of integration to be zero, without any loss of generality, we obtain

$A=-\frac{3}{4} \ln (r)-\frac{3}{4} \delta \ln [r-4 \alpha+\sqrt{(r-8 \alpha) r}]$.

When we calculate the limit $r \rightarrow+\infty$ we get $e^{2 A} \rightarrow 0$ when $\delta=+1$ and $e^{2 A} \rightarrow 2 \sqrt{2}$ when $\delta=-1$. Thus, since we must have a flat spacetime at this limit, hereinafter, we assume $\delta=-1$. Besides, we can also notice that for $\alpha>0$ the metric function (38) becomes imaginary for $r<8 \alpha$. Thus, we assert that this solution could be used as the exterior vacuum solution of a static system, where $r>r_{\Sigma}=8 \alpha$ and $r_{\Sigma}$ would be the radius of the static system. In this way we can eliminate the pathological imaginary part of the interior spacetime that has no physical meaning. The case $\alpha=0$ gives us a flat spacetime since $A=\frac{3}{4} \ln (2)$ and $B=0$. Thus, we will assume, hereinafter, $\alpha \leq 0$.

We can now obtain $A^{\prime \prime}$ just differentiating equation (37). Thus,

$A^{\prime \prime}=\frac{3}{4} \frac{[(r-4 \alpha) r+(8 \alpha-r) \sqrt{(r-8 \alpha) r}]}{\left[r^{2}(8 \alpha-r) \sqrt{(r-8 \alpha) r}\right]}$.

With the analytical solution $A^{\prime}$ we can get $B$ from Eq. (33) giving

$B=-\frac{1}{2} \ln (2)+\frac{1}{2} \ln \left(\sqrt{\frac{r}{r-8 \alpha}}+\frac{r}{r-8 \alpha}\right)$.

We can also obtain $A^{\prime \prime}$ and $B^{\prime}$ analytically. When we calculate the limit $r \rightarrow+\infty$ we get $e^{2 B} \rightarrow 1$. We obtain $B^{\prime}$ 
just differentiating equation (40), that is,

$B^{\prime}=\frac{2 \alpha[r-8 \alpha+2 \sqrt{(r-8 \alpha) r}]}{r(8 \alpha-r)[r-8 \alpha+\sqrt{(r-8 \alpha) r}]}$.

In order to check if the analytical equations for $A^{\prime}, B, A^{\prime \prime}$ and $B^{\prime}$, we substitute them into the field Eqs. (20)-(22) and we show that they are identically satisfied. Thus, we can get analytically the metric components $g_{r r}=e^{2 B}, g_{t t}=e^{2 A}$ and the Kretschmann scalar using Eq. (24). The Kretschmann scalar obtained is

$K=\frac{768 \alpha^{2} \sqrt{r-8 \alpha}\left[2 r(r-8 \alpha)^{2}+\left(384 \alpha^{2}-104 r \alpha+7 r^{2}\right) \sqrt{(r-8 \alpha) r}\right]}{\left[r^{11 / 2}(r-8 \alpha+\sqrt{(r-8 \alpha) r})^{4}\right]}$.

From the Kretschmann scalar, we can get the singularities which are at

$r_{\text {sing } 1}=0$,

$r_{\text {sing } 2}=8 \alpha$.

Since the radial coordinate is always positive, the second singularity does not exist, since $\alpha \leq 0$. So, the singularity at $r_{\text {sing } 1}$ is physical and

$$
\begin{aligned}
& \lim _{r \rightarrow r_{\text {sing1 }}^{-}} K=+\infty, \\
& \lim _{r \rightarrow r_{\text {sing } 1}^{+}} K=-\infty,
\end{aligned}
$$

which is independent of $\alpha$.

Substituting this Equation into (18) and (19) we get

$$
\begin{aligned}
& \chi^{a} \chi_{a}=-r^{-\frac{3}{2}}(r-4 \alpha+\sqrt{(r-8 \alpha) r})^{\frac{3}{2}}=0, \\
& \chi^{a} u_{a}=r^{-\frac{3}{4}}(r-4 \alpha+\sqrt{(r-8 \alpha) r})^{\frac{3}{4}}=0 .
\end{aligned}
$$

We can see easily again these equations do not have any root with $\alpha<0$, hence, there exists no horizon, neither Killing nor universal horizon.

In order to compare this result with the Schwarzschild spacetime, we will assume here, for the sake of simplicity, $\alpha=-1$. From the Schwarzschild we have

$$
\begin{aligned}
& A_{S c h}=\frac{1}{2} \ln \left(1-\frac{2 M}{r}\right), \\
& B_{S c h}=-\frac{1}{2} \ln \left(1-\frac{2 M}{r}\right),
\end{aligned}
$$

where $M$ is the mass of the particle. Substituting these metric functions into the Eq. (24) we have the well known Kretschmann scalar for the Schwarzschild metric

$$
K_{S c h}=\frac{48 M^{2}}{r^{6}} .
$$

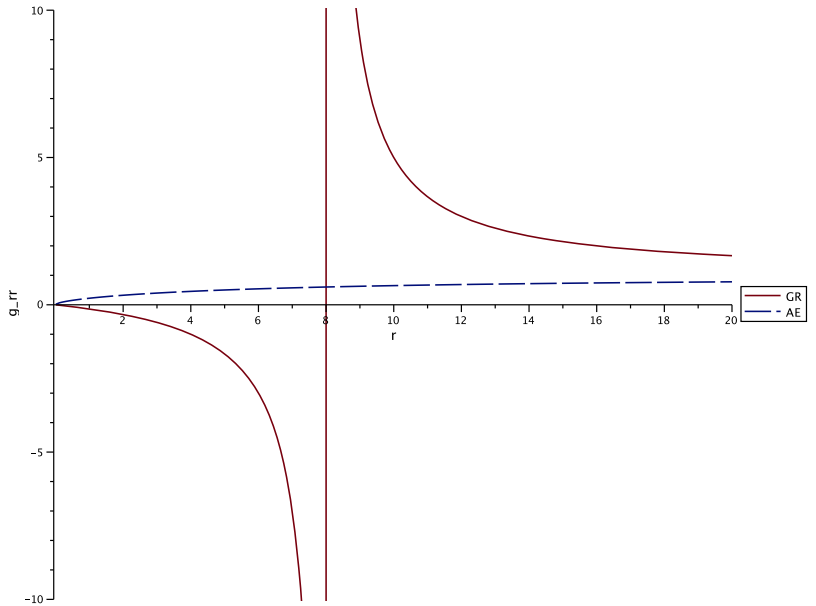

Fig. 1 Plot of the metric component $g_{r r}$, for EA parameters $c_{14}=$ $16 / 9, \alpha=-1$ and an arbitrary Schwarzschild mass $M=4$. The continuous red line represents the GR Schwarzschild metric. The dashed blue line represents the EA solution. Calculating the limit $r \rightarrow+\infty$ we obtain $g_{r r}=e^{2 B} \rightarrow 1$, as we can see in this figure. Calculating the limit $r \rightarrow r_{\text {sing } 1}=0$ we obtain $g_{r r}=e^{2 B} \rightarrow 0$, as we can see in this figure

From Eqs. (38) and (40) we get $A$ and $B$ for $\alpha=-1$. From this equation we can get $g_{r r}=e^{2 B}$ and $g_{t t}=e^{2 B}$ analytically.

$A=-\frac{3}{4} \ln (r)+\frac{3}{4} \ln [r+4+\sqrt{(r+8) r}]$,
$B=-\frac{1}{2} \ln (2)+\frac{1}{2} \ln \left(\sqrt{\frac{r}{r+8}}+\frac{r}{r+8}\right)$.

The Kretschmann scalar is obtained from Eq. (42) $K$ for $\alpha=-1$, thus

$K=\frac{768 \sqrt{r+8}\left[2 r(r+8)^{2}+\left(384+104 r+7 r^{2}\right) \sqrt{(r+8) r}\right]}{\left[r^{11 / 2}(r+8+\sqrt{(r+8) r})^{4}\right]}$.

In the Figs. 1, 2 and 3 we show the comparison of the present work quantities $g_{r r}, g_{t t}$ and $K$ with the Schwarzschild metric ones.

\subsection{Solution for $\mathbf{c}_{\mathbf{1 4}}=\mathbf{4 8} / \mathbf{2 5}$}

Solving the Eq. (31) we can obtain an analytical solution for $A^{\prime}$ for another particular case $c_{14}=48 / 25$. Making a transformation $u(r)=A^{\prime}$ we can reduce the second order differential equation in $A$ into a first order differential equation in $u$, thus

$2 r u^{\prime}+4 r u^{2}+4 u+\frac{48}{25} r^{2} u^{3}=0$. 


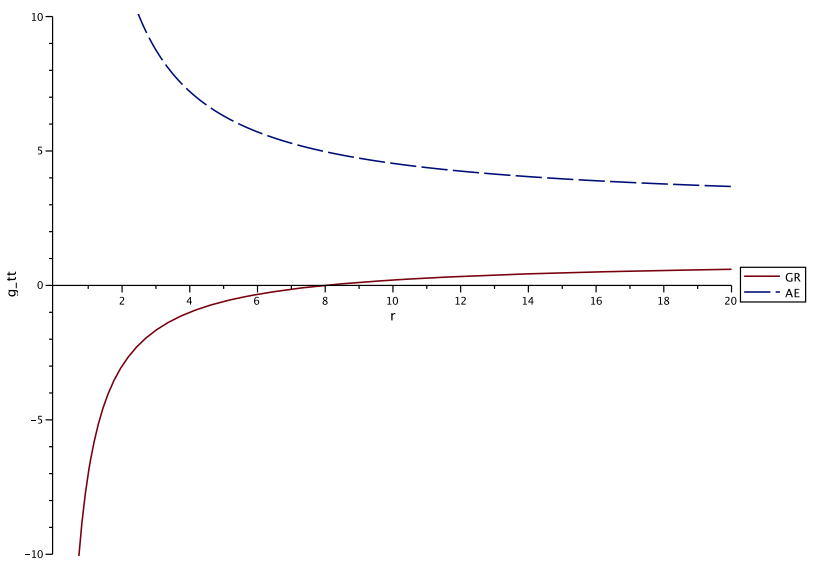

Fig. 2 Plot of the metric component $g_{t t}$, for EA parameters $c_{14}=$ $16 / 9, \alpha=-1$ and an arbitrary Schwarzschild mass $M=4$. The continuous red line represents the GR Schwarzschild metric. The dashed blue line represents the EA solution. Calculating the limit $r \rightarrow+\infty$ we obtain $g_{t t}=e^{2 A} \rightarrow 2 \sqrt{2}$, as we can see in this figure

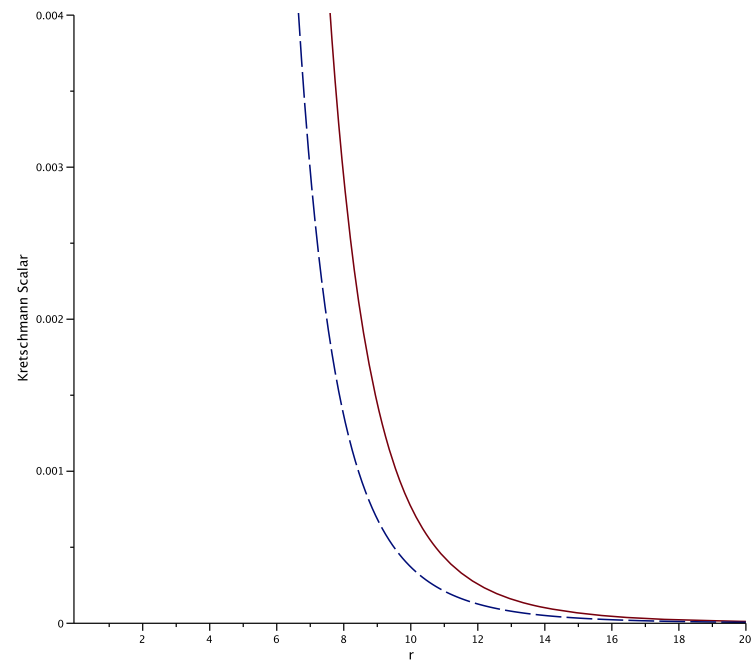

Fig. 3 Plot of the Kretschmann scalar, for EA parameters $c_{14}=16 / 9$, $\alpha=-1$ and an arbitrary Schwarzschild mass $M=4$. The continuous red line represents the GR Schwarzschild metric. The dashed blue line represents the EA solution. Calculating the limit $r \rightarrow+\infty$ we obtain $K \rightarrow 0$. Calculating the limit from left $r \rightarrow r_{\text {sing } 1}=0$ we get $K \rightarrow$ $-\infty$, as we can see in this figure. Calculating the limit from right $r \rightarrow$ $r_{\text {sing } 1}=0$ we get $K \rightarrow+\infty$, as we can see in this figure

This equation is easily integrated giving

$$
\begin{aligned}
u= & A^{\prime}=-\frac{5 \cdot 2^{\frac{1}{3}}}{12 r(-2 r+27 \gamma)} \\
& \times\left[r\left(1-3 \sqrt{3} \sqrt{\frac{\gamma}{-2 r+27 \gamma}}\right)(-2 r+27 \gamma)^{2}\right]^{\frac{1}{3}} \\
& +\frac{5 \cdot 2^{\frac{2}{3}}}{12}\left[r\left(1-3 \sqrt{3} \sqrt{\frac{\gamma}{-2 r+27 \gamma}}\right)(-2 r+27 \gamma)^{2}\right]^{-\frac{1}{3}}-\frac{5}{6 r},
\end{aligned}
$$

where $\gamma$ is an arbitrary integration constant. Substituting Eq. (52) into (33) we can calculate the limit $r \rightarrow+\infty$ we get $e^{2 B} \rightarrow 1$ only when $\gamma<0$. Thus, since we must have a flat spacetime at this limit, hereinafter, we assume $\gamma=-|\gamma|$. Thus,

$$
\begin{aligned}
u= & A^{\prime}=\frac{5}{6}\left\{-r^{-1}+(2 r)^{-\frac{2}{3}}(2 r+27|\gamma|)^{-\frac{1}{2}} \times(\sqrt{2 r+27|\gamma|}-\sqrt{27|\gamma|})^{\frac{1}{3}}\right. \\
& \left.+(2 r)^{-\frac{1}{3}}(2 r+27|\gamma|)^{-\frac{1}{2}}(\sqrt{2 r+27|\gamma|}-\sqrt{27|\gamma|})^{-\frac{1}{3}}\right\} .
\end{aligned}
$$

Substituting Eq. (53) into (33) we get

$$
\begin{aligned}
B= & -\frac{1}{3} \ln (2)-\frac{1}{2} \ln (3)+\frac{1}{2} \ln \left[2^{\frac{5}{3}} r(2 r+27|\gamma|)^{-1}\right. \\
& +2 r^{\frac{4}{3}}(2 r+27|\gamma|)^{-1}(\sqrt{27|\gamma|}-\sqrt{2 r+27|\gamma|})^{-\frac{2}{3}} \\
& -2^{\frac{1}{3}} r^{\frac{2}{3}}(2 r+27|\gamma|)^{-\frac{1}{2}}(\sqrt{27|\gamma|}-\sqrt{2 r+27|\gamma|})^{-\frac{1}{3}} \\
& +2^{\frac{1}{3}} r^{\frac{2}{3}}(2 r+27|\gamma|)^{-1}(\sqrt{27|\gamma|}-\sqrt{2 r+27|\gamma|})^{\frac{2}{3}} \\
& \left.-r^{\frac{1}{3}}(2 r+27|\gamma|)^{-\frac{1}{2}}(\sqrt{27|\gamma|}-\sqrt{2 r+27|\gamma|})^{\frac{1}{3}}\right] .
\end{aligned}
$$

Integrating the Eq. (53) using Mathematica for $|\gamma|=1$, we obtain $A$ which is given by

$$
\begin{aligned}
A= & \frac{5}{4(2 r)^{2 / 3}}(\sqrt{2 r+27}-\sqrt{27})^{4 / 3} \\
& \times{ }_{2} F_{1}\left(\frac{2}{3}, 1 ; \frac{5}{3} ; \frac{r-3 \sqrt{6 r+81}+27}{r}\right) \\
& -\frac{5}{6} \log (r)-\frac{5}{6(4 r)^{2 / 3}}(3 \sqrt{6 r+81}+27+r)^{1 / 3} \\
& \times(\sqrt{2 r+27}-\sqrt{27})^{2 / 3}[\log (\sqrt{2 r+27}-\sqrt{27}) \\
& +3 \log \left(1-\sqrt[3]{\frac{3 \sqrt{6 r+81}+27}{r}+1}\right) \\
& \left.+2 \sqrt{3} \tan ^{-1}\left(\frac{2 \sqrt[3]{\frac{3 \sqrt{6 r+81}+27}{r}+1}+1}{\sqrt{3}}\right)\right]
\end{aligned}
$$

where ${ }_{2} F_{1}(a, b ; c ; x)$ is a hypergeometric function. Substituting this Eq. into (18) and (19) we found that these equations do not have any root, hence, there exists no horizon, neither Killing nor universal. Thus, we have naked singularities.

We obtain $B^{\prime}$ just differentiating equation (40). In order to check the consistency of $A^{\prime}, B, A^{\prime \prime}$ and $B^{\prime}$, we substitute them into the field Eqs. (20)-(22) and show that they are identically satisfied. Now, we can get analytically the metric components $g_{r r}=e^{2 B}, g_{t t}=e^{2 A}$ and the Kretschmann 


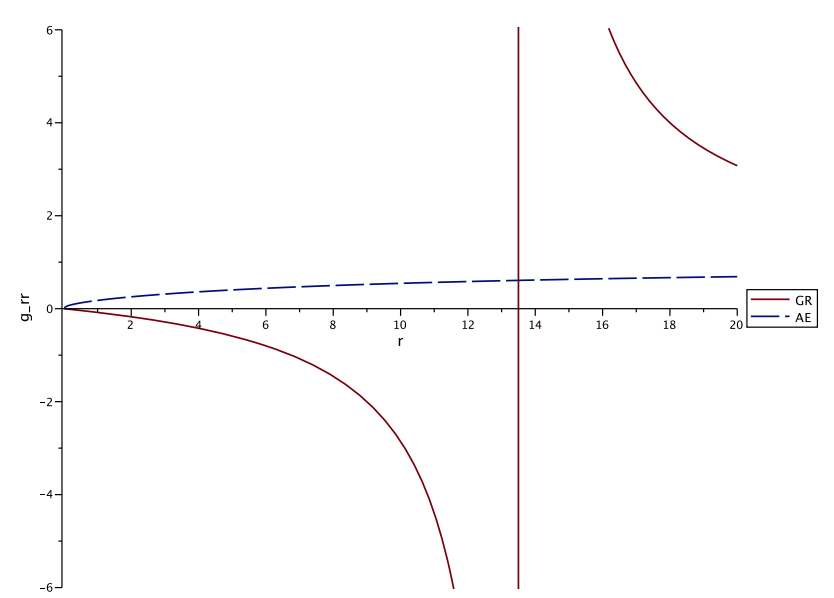

Fig. 4 Plot of the metric component $g_{r r}$, for EA parameters $c_{14}=$ $48 / 25,|\gamma|=1$ and an arbitrary Schwarzschild mass $M=27 / 4$. The continuous red line represents the GR Schwarzschild metric. The dashed blue line represents the EA solution. Calculating the limit $r \rightarrow+\infty$ we obtain $g_{r r}=e^{2 B} \rightarrow 1$, as we can see in this figure. Calculating the limit $r \rightarrow r_{\text {sing } 1}=0$ we obtain $g_{r r}=e^{2 B} \rightarrow 0$, as we can see in this figure

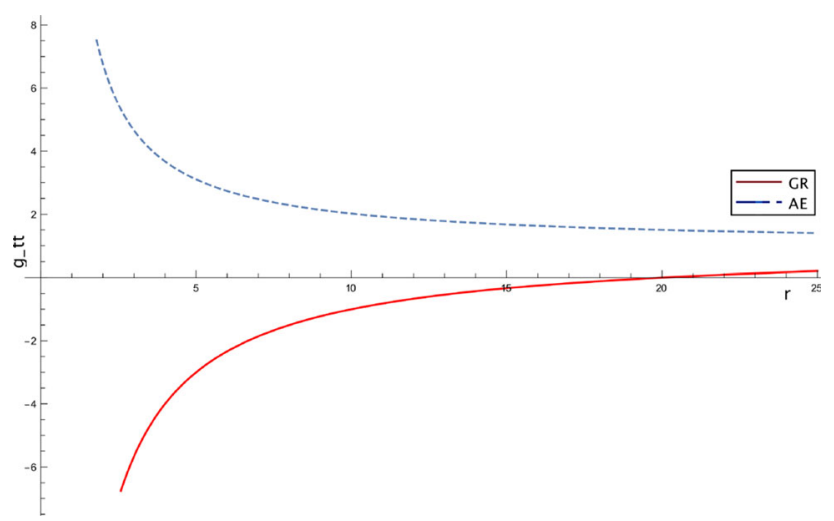

Fig. 5 Plot of the metric component $g_{t t}$, for EA parameters $c_{14}=$ $48 / 25,|\gamma|=1$ and an arbitrary Schwarzschild mass $M=10$ The continuous red line represents the GR Schwarzschild metric. The dashed blue line represents the EA solution. Calculating the limit $r \rightarrow+\infty$, we obtain $g_{t t}=e^{2 A} \rightarrow 1$, as we can see in this figure. Calculating the limit from left $r \rightarrow r_{\text {sing } 1}=0$ we obtain $g_{t t}=e^{2 A} \rightarrow+\infty$, as we can see in this figure

scalar using Eq. (24). The Kretschmann scalar is calculated but not shown because it is too long. From the Kretschmann scalar we can get the singularity which are given by

$r_{\text {sing } 1}=0$.

In order to compare this result with the Schwarzschild spacetime, we use the Eqs. (47) and (48). For the sake of simplicity we assume also $|\gamma|=1$. The Kretschmann scalar is obtained from Eq. (24) analytically. In the Figs. 4, 5, and 6 we show the comparison of the present work quantities $g_{r r}$, $g_{t t}$ and $K$ with the Schwarzschild metric ones.

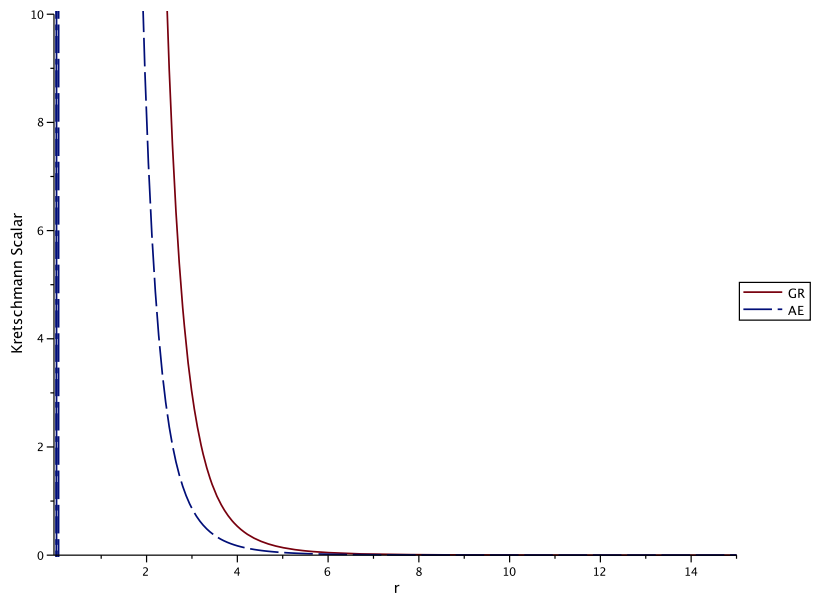

Fig. 6 Plot of the Kretschmann scalar, for EA parameters $c_{14}=48 / 25$, $|\gamma|=1$ and an arbitrary Schwarzschild mass $M=27 / 2$. The continuous red line represents the GR Schwarzschild metric. The dashed blue line represents the EA solution. Calculating the limit $r \rightarrow+\infty$ we obtain $K \rightarrow 0$. Calculating the limit from left $r \rightarrow r_{\text {sing } 1}=0$ we obtain $K \rightarrow-\infty$, as we can see in this figure. Calculating the limit from right $r \rightarrow r_{\text {sing } 1}=0$ we obtain $K \rightarrow+\infty$, as we can see in this figure

\subsection{Solution for $\mathbf{c}_{\mathbf{1 4}}<\mathbf{0}$}

Taking $c_{14}<0$ implies that the coupling constant of EA theory is stronger than the Newtonian gravitational constant [see Eq. (5)]. Although this possibility is ruled out by experimental data [51], we include this case just for the sake of completeness. Solving the Eq. (31) we can obtain an analytical solution for $A^{\prime}$ for another particular case $c_{14}=-16$. Making a transformation $u(r)=A^{\prime}$ we can reduce the second order differential equation in $A$ into a first order differential equation in $u$, thus

$2 r u^{\prime}+4 r u^{2}+4 u-16 r^{2} u^{3}=0$.

This equation is easily integrated giving

$$
u=A^{\prime}=\frac{\kappa\left[\left(4+4 r \sqrt{\frac{r}{-32 \kappa^{3}+r^{3}}}\right)^{\frac{2}{3}}\left(32 \kappa^{3}-r^{3}\right)^{\frac{1}{3}}+8 \kappa\right]}{2 r\left(32 \kappa^{3}-r^{3}\right)^{\frac{2}{3}}\left(4+4 r \sqrt{\frac{r}{-32 \kappa^{3}+r^{3}}}\right)^{\frac{1}{3}}},
$$

where $\kappa$ is an arbitrary integration constant. The solution with $\kappa>0$ give us an imaginary solution, thus it will not be considered here. The solution with $\kappa<0$ is gives us real solution. Thus in the following equation we assume that $\kappa=-|\kappa|$.

$$
\begin{aligned}
u= & A^{\prime}=\frac{2^{\frac{5}{9}}|\kappa|\left(\sqrt{32|\kappa|^{3}+r^{3}}+\sqrt{r^{3}}\right)^{\frac{1}{3}}}{2 r \sqrt{32|\kappa|^{3}+r^{3}}} \\
& +\frac{2|\kappa|}{r \sqrt{32|\kappa|^{3}+r^{3}}\left(\sqrt{32|\kappa|^{3}+r^{3}}+\sqrt{r^{3}}\right)^{\frac{1}{3}}}
\end{aligned}
$$


Substituting Eq. (59) into (33) we can calculate the limit $r \rightarrow+\infty$ we get $e^{2 B} \rightarrow 1$ only when $\kappa<0$. Thus, we have a flat spacetime at this limit. Thus,

$$
\begin{aligned}
& B=\frac{1}{3} \ln (2)+\frac{1}{2} \ln \left\{r\left\{2048 \sqrt{\frac{r}{32|\kappa|^{3}+r^{3}}}\right)|\kappa|^{7}\right. \\
& -128.2^{\frac{2}{3}}|\kappa|^{5}\left[\left(1+r \sqrt{\frac{r}{32|\kappa|^{3}+r^{3}}}\right)\left(32|\kappa|^{3}+r^{3}\right)^{2}\right]^{\frac{1}{3}} \\
& \left.\times \sqrt{\frac{r}{32|\kappa|^{3}+r^{3}}}+128|\kappa|^{4} r^{3} \sqrt{\frac{r}{32|\kappa|^{3}+r^{3}}}\right)+64|\kappa|^{4} r^{2} \\
& -4.2^{\frac{2}{3}}|\kappa|^{2} r^{3}\left[\left(1+r \sqrt{\frac{r}{32|\kappa|^{3}+r^{3}}}\right)\left(32|\kappa|^{3}+r^{3}\right)^{2}\right]^{\frac{1}{3}} \\
& \left.\times \sqrt{\frac{r}{32|\kappa|^{3}+r^{3}}}+2|\kappa| r^{6} \sqrt{\frac{r}{32|\kappa|^{3}+r^{3}}}\right)+2|\kappa| r^{5} \\
& \left.+2^{\frac{1}{3}} r^{2}\left[\left(1+r \sqrt{\frac{r}{32|\kappa|^{3}+r^{3}}}\right)\left(32|\kappa|^{3}+r^{3}\right)^{2}\right]^{\frac{2}{3}}\right\} \\
& \times\left[\left(4|\kappa|+2^{\frac{1}{3}} r\right)\left(16|\kappa|^{2}-42^{\frac{1}{3}}|\kappa| r+2^{\frac{2}{3}} r^{2}\right)\right. \\
& \left.\left.\times\left[\left(1+r \sqrt{\frac{r}{32|\kappa|^{3}+r^{3}}}\right)\left(32|\kappa|^{3}+r^{3}\right)^{2}\right]^{\frac{2}{3}}\right]^{-1}\right\} .
\end{aligned}
$$

Integrating Eq. (59) using Mathematica for $|\kappa|=1$, we obtain

$$
\begin{aligned}
A= & \frac{1}{962^{4 / 9}}\left[-3\left(r^{3 / 2}+\sqrt{r^{3}+32}\right)^{4 / 3}\right. \\
& \times{ }_{2} F_{1}\left(\frac{2}{3}, 1 ; \frac{5}{3} ; \frac{1}{16}\left(r^{3}+\sqrt{r^{3}+32} r^{3 / 2}+16\right)\right) \\
& -8 \sqrt[9]{2}\left(-2 \log \left(22^{2 / 3}-\left(r^{3 / 2}+\sqrt{r^{3}+32}\right)^{2 / 3}\right)\right. \\
& +\log \left(\left(r^{3 / 2}+\sqrt{r^{3}+32}\right)^{4 / 3}+22^{2 / 3}\left(r^{3 / 2}+\sqrt{r^{3}+32}\right)^{2 / 3}\right. \\
& +8 \sqrt[3]{2}) \\
& \left.+2 \sqrt{3} \tan ^{-1}\left(\frac{\left(r^{3 / 2}+\sqrt{r^{3}+32}\right)^{2 / 3}+2^{2 / 3}}{2^{2 / 3} \sqrt{3}}\right)\right]
\end{aligned}
$$

where ${ }_{2} F_{1}(a, b ; c ; x)$ is a hypergeometric function. Substituting this equation into (18) and (19) we found that these equations do not have any root, hence, there exists no horizon, neither Killing nor universal. Thus, we have naked singularities. In the Figs. 7, 8, and 9 we show the comparison of the present work quantities $g_{r r}, g_{t t}$ and $K$ with the Schwarzschild metric ones.

\section{Solutions for $\mathrm{c}_{14}=0$}

Taking $c_{14}=0$ implies that the coupling constant of EA theory is the same as the Newtonian gravitational constant

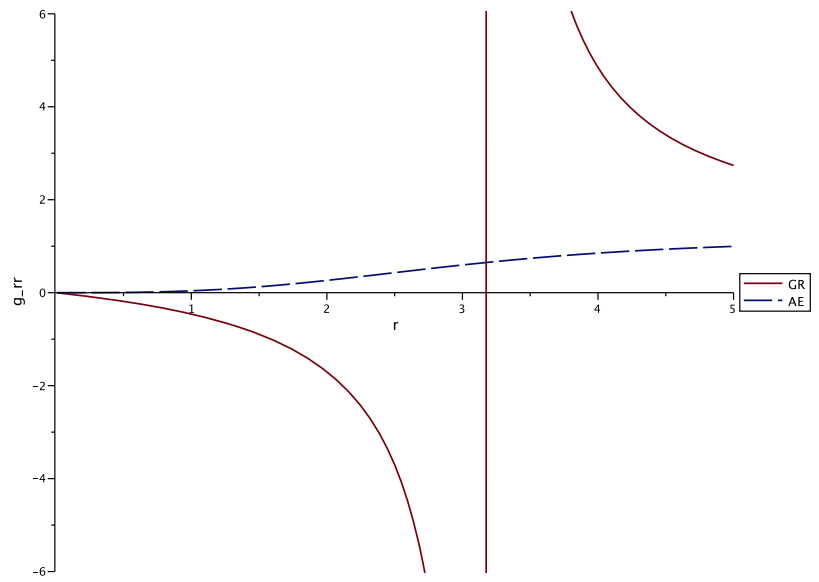

Fig. 7 Plot of the metric component $g_{r r}$, for EA parameters $c_{14}=$ $-16,|\kappa|=1$ and an arbitrary Schwarzschild mass $M=32^{\frac{1}{3}} / 2$. The continuous red line represents the GR Schwarzschild metric. The dashed blue line represents the EA solution. Calculating the limit $r \rightarrow+\infty$ we obtain $g_{r r}=e^{2 B} \rightarrow 1$, as we can see in this figure. Calculating the limit $r \rightarrow r_{\text {sing } 1}=0$ we obtain $g_{r r}=e^{2 B} \rightarrow 0$, as we can see in this figure

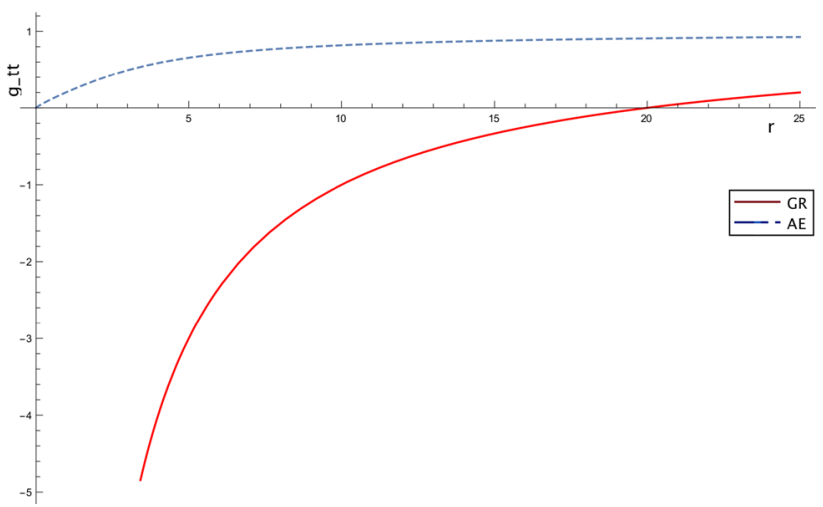

Fig. 8 Plot of the metric component $g_{t t}$, for EA parameters $c_{14}=-16$, $|\kappa|=1$ and an arbitrary Schwarzschild mass $M=10$. The continuous red line represents the GR Schwarzschild metric. The dashed blue line represents the EA solution. Calculating the limit $r \rightarrow+\infty$, we obtain $g_{t t}=e^{2 A} \rightarrow 1$, as we can see in this figure. Calculating the limit from right $r \rightarrow r_{\text {sing } 1}=0$ we obtain $g_{t t}=e^{2 A} \rightarrow 0$, as we can see in this figure

G. Substituting $c_{14}=0$ into the field Eqs. (20)-(22), we get

$$
\begin{aligned}
& 2 r B^{\prime}+e^{2 B}-1=0, \\
& 2 r A^{\prime}-e^{2 B}+1=0, \\
& r A^{\prime \prime}-r A^{\prime} B^{\prime}+r A^{\prime 2}+A^{\prime}-B^{\prime}=0 .
\end{aligned}
$$

From the above, we have the solution

$$
A=\frac{1}{2} \ln \left(2-\frac{2 v}{r}\right)
$$




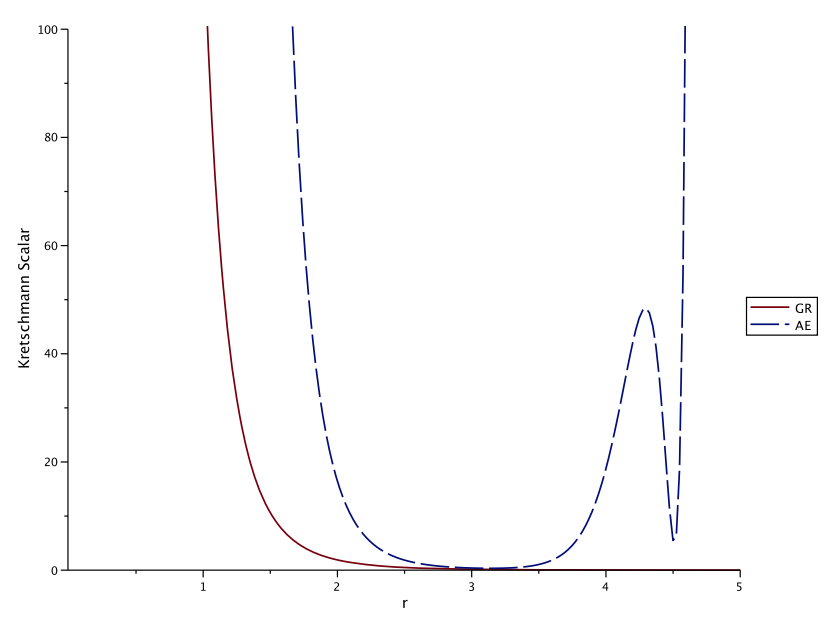

Fig. 9 Plot of the Kretschmann scalar, for EA parameters $c_{14}=-16$, $|\kappa|=1$ and an arbitrary Schwarzschild mass $M=32^{\frac{1}{3}} / 2$. The continuous red line represents the GR Schwarzschild metric. The dashed blue line represents the EA solution. Calculating the limit $r \rightarrow+\infty$ we obtain $K \rightarrow+\infty$, as we can see in this figure. Calculating the limit from left $r \rightarrow r_{\text {sing } 1}=0$ we obtain $K \rightarrow+\infty$, as we can see in this figure

and

$B=-\frac{1}{2} \ln \left(1-\frac{v}{\mu r}\right)$.

where $\mu$ and $\nu$ are some constants of integration which can be chosen appropriately. With this solution for $A$ and $B$, we can cast the metric (13) in the Schwarzschild form, that is,

$$
\begin{aligned}
d s^{2}= & -2\left(\mu-\frac{v}{r}\right) d t^{2} \\
& +\mu\left(\mu-\frac{v}{r}\right)^{-1} d r^{2}+r^{2} d \theta^{2}+r^{2} \sin ^{2} \theta d \phi^{2},
\end{aligned}
$$

which yields the standard Schwarzschild metric of GR when $\mu=1 / 2$ and $v=M$.

Substituting $A$ and $B$ equations into (18) and (19) we get

$\chi^{a} \chi_{a}=-1+\frac{2 M}{r}=0$,

which means the Killing horizon is at $r_{K H}=2 M$, and

$\chi^{a} u_{a}=\sqrt{1-\frac{2 M}{r}}=0$,

implies the universal horizon is at $r_{U H}=2 M$. We can see again that the Killing and the universal horizons coincide just as in GR.

\section{A note on black hole thermodynamics}

Although the central purpose of this article is not thermodynamics of black holes, we briefly discuss it for the sake of completeness. The essential ingredient for this is the existence of horizons, the usual event horizon in the relativistic case and universal horizon in the Lorentz-violating theories like the one we are considering here. There exists both types of horizons only in $c_{14}=0$ case of all the solutions we have found here. For more discussion on black hole thermodynaimcs in EA theory, we refer the readers to references [10, 18,52-54].

Recently, the thermodynamics of black holes in EinsteinAether-Maxwell Theory was investigated by the solution phase space method in [54]. Following their result we can directly write down the surface gravity, temperature, entropy and the first law for the Killing horizon respectively as

$$
\begin{aligned}
\kappa_{\mathrm{KH}} & =\frac{2 r_{\mathrm{KH}}-3 M}{r_{\mathrm{KH}}^{2}}, \\
T_{\mathrm{KH}} & =\frac{\kappa_{\mathrm{KH}}}{2 \pi}, \\
S_{\mathrm{KH}} & =\frac{\pi r_{\mathrm{KH}}^{2}}{G}=\frac{\mathrm{A}_{\mathrm{KH}}}{4 G}, \\
\delta S_{\mathrm{KH}} & =\frac{\delta M}{T_{\mathrm{KH}}} .
\end{aligned}
$$

Note that these expressions are straightforward to obtain from [54] simply by taking the electric charge of Einstein-AetherMaxwell black hole to be zero. Similarly, we can write down the the surface gravity, temperature, entropy and the first law for the universal horizon respectively as

$$
\begin{aligned}
\kappa_{\mathrm{UH}} & =\frac{2 r_{\mathrm{UH}}-3 M}{r_{\mathrm{UH}}^{2}}, \\
T_{\mathrm{UH}} & =\frac{\kappa_{\mathrm{UH}}}{2 \pi}, \\
S_{\mathrm{UH}} & =\frac{\pi r_{\mathrm{UH}}^{2}}{G}=\frac{\mathrm{A}_{\mathrm{UH}}}{4 G}, \\
\delta S_{\mathrm{UH}} & =\frac{\delta M}{T_{\mathrm{UH}}} .
\end{aligned}
$$

we can see that the entropies are exactly one quarter of the horizon areas, and the first laws of black hole thermodynamics are obtained both at $\mathrm{KH}$ and $\mathrm{UH}$.

As we found out, in the $c_{14}=0$ case, the Killing and the universal horizons coincide just as in GR. This is clearly reflected in the above expressions. Substituting our results $r_{K H}=r_{U H}=2 M$, we obtain the following.

$$
\begin{aligned}
& \kappa_{\mathrm{KH}}=\kappa_{\mathrm{UH}}=\frac{1}{4 M}, \\
& T_{\mathrm{KH}}=T_{\mathrm{UH}}=\frac{1}{8 \pi M},
\end{aligned}
$$




$$
\begin{aligned}
& S_{\mathrm{KH}}=S_{\mathrm{UH}}=\frac{4 \pi M^{2}}{G} \\
& \delta S_{\mathrm{KH}}=\delta S_{\mathrm{UH}}=8 \pi M \delta M .
\end{aligned}
$$

It is clear from the equations above that they are exactly the same as in GR. However, for a non-static aether, one may have to redefine surface gravity because of the contribution of aether vector field [54].

\section{Conclusions}

In the present work, we have analyzed all the possible exterior vacuum solutions with spherical symmetry allowed by the EA theory with static aether. We show that there are three classes of explicit analytic solutions corresponding to different values for $c_{14}$, which are: $0<c_{14}<2, c_{14}<0$ and $c_{14}=0$. Our results are summarized below.

(i) For $0<c_{14}<2$ we present analytical solutions for $c_{14}=3 / 2, c_{14}=16 / 9$ and $c_{14}=48 / 25$. The $c_{14}=3 / 2$ solution is excluded because the spacetime becomes imaginary at a finite radius. The other two analytical solutions $\left(c_{14}=16 / 9\right.$ and $\left.48 / 25\right)$ have singularities at $r=0$, and are asymptotically flat spacetimes as in the case of Schwarzschild metric in GR. These solutions have no horizons, neither Killing nor universal. This suggests that we can have naked singularities.

(ii) For $c_{14}<0$, the absence of any horizons again suggests the presence of a naked singularity at the origin and the spacetime is asymptotically flat.

(iii) For $c_{14}=0$ we have the Schwarzschild spacetime, which also picks Newtonian gravitational constant as coupling. As expected, the Killing horizon coincides with the universal horizon and is the same as the event horizon of the Schwarzschild spacetime in GR.

In the 2006 paper, Eling and Jacobson [8] analyzed the behavior of the static aether solutions for $0 \leq c_{14}<2$. They asserted that regular black holes cannot have static aether fields since the Killing vector is null, not timelike on the horizon as always is the case with static aether. As far as we know, this the only work in EA theory that mentions the existence of naked singularity. What we show here is the corollary, meaning the static aether leads to absence of any kind of horizon, suggesting a possible formation of naked singularities, which could circumvent the need for switching from timelike vector to null vector at the horizon by totally avoiding the existence of the horizon itself. Of course, to rigorously prove that the singularity is naked, it would be still necessary to prove that future directed null geodesics can emanate from the singularity.
Finally, we note that the unique case where we have black hole is for $c_{14}=0$ which furnishes the GR limit. For this the gravitational mass is the same as the Schwarzschild mass [see Eq. (65)]. We have calculated the Killing and the universal horizons and studied their thermodynamic properties. Besides, for the other solutions $\left(c_{14}=16 / 9,48 / 25\right.$ and $\left.c_{14}<0\right)$ we found that there are no horizons, neither Killing nor universal. This suggests that we can have naked singularities.

Acknowledgements The author (RC) acknowledges the financial support from FAPERJ (no.E-26/171.754/2000, E-26/171.533/2002 and E-26/170.951/2006). MFAdaS acknowledges the financial support from CNPq-Brazil, FINEP-Brazil (Ref. 2399/03), FAPERJ/UERJ (307935/2018-3) and from CAPES (CAPES-PRINT 41/2017). VHS thanks José Abdalla Helayäl-Neto for hospitality at Centro Brasileiro de Pesquisas Físicas (CBPF). The authors are grateful to Ted Jacobson for pointing out errors in the first version.

Data Availability Statement This manuscript has no associated data or the data will not be deposited. [Authors' comment: This paper is a pure theoretical one, thus we do not need nor supplementary observational neither laboratorial data.]

Open Access This article is licensed under a Creative Commons Attribution 4.0 International License, which permits use, sharing, adaptation, distribution and reproduction in any medium or format, as long as you give appropriate credit to the original author(s) and the source, provide a link to the Creative Commons licence, and indicate if changes were made. The images or other third party material in this article are included in the article's Creative Commons licence, unless indicated otherwise in a credit line to the material. If material is not included in the article's Creative Commons licence and your intended use is not permitted by statutory regulation or exceeds the permitted use, you will need to obtain permission directly from the copyright holder. To view a copy of this licence, visit http://creativecomm ons.org/licenses/by/4.0/.

Funded by $\mathrm{SCOAP}^{3}$.

\section{References}

1. D.G. Moore, V.H. Satheeshkumar, Int. J. Mod. Phys. D 22, 1342026 (2013). arXiv:1305.7221 [gr-qc]

2. H. Pihan-Le Bars et al., Phys. Rev. Lett. 123(23), 231102 (2019). arXiv:1912.03030 [physics.space-ph]

3. T. Jacobson, D. Mattingly, Phys. Rev. D 64, 024028 (2001). arXiv:gr-qc/0007031

4. C. Eling, T. Jacobson, Phys. Rev. D 69, 064005 (2004). arXiv:gr-qc/0310044

5. T. Jacobson, D. Mattingly, Phys. Rev. D 70, 024003 (2004). arXiv:gr-qc/0402005

6. C. Eling, T. Jacobson, D. Mattingly, in Deserfest: A celebration of the life and works of Stanley Deser. Proceedings, Meeting, Ann Arbor, USA, April 3-5, 2004, ed. by J.T. Liu, M.J. Duff, K.S. Stelle, R.P. Woodard. arXiv:gr-qc/0410001

7. B.Z. Foster, T. Jacobson, Phys. Rev. D 73, 064015 (2006). arXiv:gr-qc/0509083

8. C. Eling, T. Jacobson, Class. Quantum Gravit. 23, 5643 (2006). arXiv:gr-qc/0604088 [Erratum: Class. Quant. Grav. 27, 049802 (2010)] 
9. C. Eling, T. Jacobson, M. Coleman Miller, Phys. Rev. D 76, 042003 (2007). arXiv:0705.1565 [gr-qc] [Erratum: Phys. Rev. D 80, 129906 (2009)]

10. B.Z. Foster, Phys. Rev. D 73, 024005 (2006). arXiv:gr-qc/0509121

11. D. Garfinkle, C. Eling, T. Jacobson, Phys. Rev. D 76, 024003 (2007). arXiv:gr-qc/0703093 [gr-qc]

12. R.A. Konoplya, A. Zhidenko, Phys. Lett. B 644, 186 (2007). arXiv:gr-qc/0605082

13. T. Tamaki, U. Miyamoto, Phys. Rev. D 77, 024026 (2008). arXiv:0709.1011 [gr-qc]

14. E. Barausse, T. Jacobson, T.P. Sotiriou, Phys. Rev. D 83, 124043 (2011). arXiv:1104.2889 [gr-qc]

15. C. Gao, Y.G. Shen, Phys. Rev. D 88, 103508 (2013). arXiv:1301.7122 [gr-qc]

16. C. Ding, A. Wang, X. Wang, Phys. Rev. D 92(8), 084055 (2015). arXiv:1507.06618 [gr-qc]

17. C. Ding, C. Liu, A. Wang, J. Jing, Phys. Rev. D 94(12), 124034 (2016). arXiv:1608.00290 [gr-qc]

18. C. Ding, A. Wang, X. Wang, T. Zhu, Nucl. Phys. B 913, 694 (2016). arXiv:1512.01900 [gr-qc]

19. E. Barausse, T.P. Sotiriou, I. Vega, Phys. Rev. D 93(4), 044044 (2016). arXiv:1512.05894 [gr-qc]

20. J. Latta, G. Leon, A. Paliathanasis, JCAP 1611, 051 (2016). arXiv: 1606.08586 [gr-qc]

21. K. Lin, F.H. Ho, W.L. Qian, Int. J. Mod. Phys. D 28(03), 1950049 (2018). arXiv:1704.06728 [gr-qc]

22. C. Ding, Phys. Rev. D 96(10), 104021 (2017). arXiv:1707.06747 [gr-qc]

23. M. Bhattacharjee, S. Mukohyama, M.B. Wan, A. Wang, Phys. Rev. D 98(6), 064010 (2018). arXiv:1806.00142 [gr-qc]

24. K. Lin et al., Phys. Rev. D 99(2), 023010 (2019). arXiv: 1810.07707 [astro-ph]

25. T. Zhu, Q. Wu, M. Jamil, K. Jusufi, Phys. Rev. D 100(4), 044055 (2019). arXiv:1906.05673 [gr-qc]

26. C. Ding, Nucl. Phys. B 938, 736 (2019). arXiv:1812.07994 [gr-qc]

27. A. Coley, G. Leon, Gen. Relativ. Gravit. 51(9), 115 (2019). arXiv: 1905.02003 [gr-qc]

28. G. Leon, A. Coley, A. Paliathanasis, Ann. Phys. 412, 168002 (2020). arXiv:1906.05749 [gr-qc]

29. C. Zhang, X. Zhao, A. Wang, B. Wang, K. Yagi, N. Yunes, W. Zhao and T. Zhu, Phys. Rev. D 101(4), 044002 (2020). arXiv:1911.10278 [gr-qc]

30. C. Zhang, X. Zhao, K. Lin, S. Zhang, W. Zhao, A. Wang, (2020). arXiv:2004.06155 [gr-qc]

31. R. Penrose, Phys. Rev. Lett. 14, 57-59 (1965)

32. The Nobel Prize in Physics 2020. NobelPrize.org. Nobel Media AB 2020. Sun. 11 Oct 2020 (2020). https://www.nobelprize.org/ prizes/physics/2020/summary/

33. R. Penrose, Riv. Nuovo Cim. 1, 252-276 (1969)
34. R. Penrose, in Black holes and relativistic stars, ed. by R.M. Wald (University of Chicago Press, Chicago, 1998)

35. A. Krolak, Prog. Theor. Phys. Suppl. 136, 45-56 (1999). arXiv:gr-qc/9910108

36. P.S. Joshi, Pramana 55, 529-544 (2000). arXiv:gr-qc/0006101

37. M.N. Celerier, P. Szekeres, Phys. Rev. D 65, 123516 (2002). arXiv:gr-qc/0203094

38. R. Giambo, F. Giannoni, G. Magli, P. Piccione, Commun. Math. Phys. 235, 545-563 (2003). arXiv:gr-qc/0204030

39. T. Harada, H. Iguchi, K.I. Nakao, Prog. Theor. Phys. 107, 449-524 (2002). arXiv:gr-qc/0204008

40. C.F.C. Brandt, L.M. Lin, J.F. Villas da Rocha, A.Z. Wang, Int. J. Mod. Phys. D 11, 155-186 (2002). arXiv:gr-qc/0105019

41. R. Chan, M.F.A. da Silva, J.F. Villas da Rocha, Int. J. Mod. Phys. D 12, 347-368 (2003). arXiv:gr-qc/0209067

42. P.S. Joshi, The Story of Collapsing Stars: Black Holes, Naked Singularities, and the Cosmic Play of Quantum Gravity (Oxford University Press, Oxford, 2015)

43. P.S. Joshi, D. Malafarina, R. Narayan, Class. Quantum Gravit. 31, 015002 (2014). arXiv:1304.7331 [gr-qc]

44. C. Chakraborty, P. Kocherlakota, P.S. Joshi, Phys. Rev. D 95(4), 044006 (2017). arXiv:1605.00600 [gr-qc]

45. C. Chakraborty, M. Patil, P. Kocherlakota, S. Bhattacharyya, P.S. Joshi, A. Królak, Phys. Rev. D 95(8), 084024 (2017). arXiv: 1611.08808 [gr-qc]

46. C. Chakraborty, S. Bhattacharyya, Phys. Rev. D 98(4), 043021 (2018). arXiv:1712.01156 [astro-ph.HE]

47. C. Chakraborty, S. Bhattacharyya, JCAP 05, 034 (2019). arXiv:1901.04233 [astro-ph.HE]

48. R. Shaikh, P.S. Joshi, JCAP 10, 064 (2019). arXiv:1909.10322 [grqc]

49. K. Lin, O. Goldoni, M.F. da Silva, A. Wang, Phys. Rev. D 91(2), 024047 (2015). arXiv:1410.6678 [gr-qc]

50. P. Berglund, J. Bhattacharyya, D. Mattingly, Phys. Rev. D 85, 124019 (2012). arXiv:1202.4497 [hep-th]

51. J. Oost, S. Mukohyama, A. Wang, Phys. Rev. D 97(12), 124023 (2018). arXiv:1802.04303 [gr-qc]

52. P. Berglund, J. Bhattacharyya, D. Mattingly, Phys. Rev. Lett. 110(7), 071301 (2013). arXiv:1210.4940 [hep-th]

53. C. Ding and A. Wang, Phys. Rev. D 99(12), 124011 (2019). arXiv: 1811.05779 [gr-qc]

54. H.F. Ding, X.H. Zhai, Class. Quantum Gravit. 37(18), 185015 (2020). arXiv:2001.06261 [gr-qc] 